Revista Brasil. Bot., V.33, n.1, p.85-91, jan.-mar. 2010

\title{
Estudo dos anéis de crescimento de Schizolobium parahyba (Leguminosae: Caesalpinioideae) após episódio de mortalidade em Ilha Grande, Rio de Janeiro
}

\author{
CÁTIA HENRIQUES CALLADO ${ }^{1,2}$ e ROSANA CARDOSO GUIMARÃES ${ }^{1}$
}

(recebido: 12 de fevereiro de 2009; aceito: 16 de dezembro de 2009)

\begin{abstract}
Tree-ring study of Schizolobium parahyba (Leguminosae: Caesalpinioideae) after a mortality episode in Ilha Grande, Rio de Janeiro state). Schizolobium parahyba (Vell.) S.F. Blake is a native species of the Atlantic rain forest which trees disappeared from Ilha Grande, Rio de Janeiro, due to a mortality episode. In this study, the growth-rings were investigated with the objective of determining the age of the dead trees, the relationship between growth-ring width and local precipitation, and the possible relationship between mortality episode and the population age structure or successional processes. The results showed that dead individuals had different ages and that growth-rings width was significantly correlated with the annual precipitation in the period investigated. The possibility of the death episode being part of a natural succession process or a particular event of a senescent population was discarded and it is suggested that climatic anomalies that occurred between the years 1997 and 2001 have probably contributed to tree mortality.
\end{abstract}

Keywords - Brazil, guapuruvu, tree-growth analysis, tropical rain forest

RESUMO - (Estudo dos anéis de crescimento de Schizolobium parahyba (Leguminosae: Caesalpinioideae) após episódio de mortalidade em Ilha Grande, Rio de Janeiro). Schizolobium parahyba (Vell.) S.F. Blake é uma espécie nativa da Floresta Atlântica, cujos indivíduos arbóreos desapareceram devido a um episódio de mortalidade na Ilha Grande, Rio de Janeiro. Neste trabalho, os anéis de crescimento foram investigados com o objetivo de determinar a idade das árvores mortas, a relação da largura desses anéis com os índices pluviométricos locais e a possível relação do episódio de mortalidade com a estrutura etária da população ou com processos sucessionais. Os resultados demonstraram que os indivíduos morreram com diferentes idades e que a largura dos anéis de crescimento foi significativamente correlacionada com a precipitação anual no período investigado. A possibilidade do episódio de mortalidade refletir um processo de sucessão natural ou um evento particular de uma população senescente foi descartada e é provável que as anomalias climáticas ocorridas entre os anos de 1997 e 2001 tenham contribuído para a mortalidade das árvores.

Palavras-chave - Brasil, crescimento de árvores, floresta tropical, guapuruvu

\section{Introdução}

Schizolobium parahyba (Vell.) S. F. Blake (Leguminosae: Caesalpinioideae), o guapuruvu ou bacurubu, é uma espécie nativa da Floresta Atlântica, cuja área natural de distribuição compreende a vertente litorânea da Serra do Mar, desde o Rio de Janeiro até o Rio Grande do Sul (Pio Corrêa 1926, Richter et al. 1974, Lorenzi 1992, Rizzini 1995, Sousa et al. 2005).

A história de $S$. parahyba na Ilha Grande, Município de Angra dos Reis, Estado do Rio de Janeiro, se confunde com a dos habitantes locais. Os caiçaras tinham por hábito plantar árvores dessa espécie para confecção de canoas para pesca (Santos et al. 2009). Isso se deve à baixa densidade de sua madeira e as altas taxas de

\footnotetext{
1. Universidade do Estado do Rio de Janeiro, Instituto de Biologia Roberto Alcantara Gomes, Departamento de Biologia Vegetal, Rua São Francisco Xavier, 524, PHLC, sala 224, 20550-013 Rio de Janeiro, RJ, Brasil.

2._Autor para correspondência: ccallado@uerj.br
}

crescimento radial (Richter et al. 1974, Ferreira 2002, Lisi et al. 2008, Marcati et al. 2008). Possivelmente, $S$. parahyba é uma das essências florestais de mais rápido crescimento nas regiões sudeste e sul do Brasil (Inoue et al. 1984). Até alguns poucos anos, o trabalho direto ou indireto na pesca era uma das principais atividades sócioeconômicas desenvolvidas na Ilha Grande. Todavia, a redução dos recursos pesqueiros, associada à criação de unidades de conservação, o que impediu o corte seletivo de árvores a partir de 1971 (Santos et al. 2009), possibilitaram o aumento da população de $S$. parahyba na região. Essa situação permaneceu até o final do ano de 2001, quando foi constatada a mortalidade de todos os indivíduos arbóreos da espécie.

$\mathrm{O}$ ambiente insular pode provocar um parentesco muito próximo entre os indivíduos de uma população em atividade reprodutiva e essa estruturação familiar conduz à perda de variabilidade genética (Ellstrand \& Elam 1993). Contudo, o estudo de Freire et al. (2007) constatou que a diversidade genética da população de S. parahyba na Ilha Grande era superior a de outras 
populações localizadas em diferentes áreas do continente. Esse resultado descartou a hipótese de que problemas relacionados à baixa variabilidade genética teriam causado enfraquecimento e morte dos indivíduos da espécie na ilha.

A identificação das taxas e das causas de mortalidade de espécies arbóreas é essencial, pois permite predizer aspectos relativos ao crescimento das florestas e entender possíveis respostas a situações de estresse ambiental. Compreender esses aspectos da dinâmica populacional em ecossistemas naturais tem se tornado uma ação cada vez mais relevante, visto que muitas espécies têm declinado em direção à extinção (Ricklefs 2003). Cabe ressaltar que os fatores responsáveis pela mortalidade natural são ainda muito pouco conhecidos (Franklin et al. 1987), principalmente quando se trata da mortalidade de uma única espécie em uma floresta aparentemente saudável (Harcombe \& Marks 1983).

Vários fatores podem causar a mortalidade de espécies vegetais. Esses são definidos como (i) regulares, quando estão associados a competição por água, luz e nutrientes do solo, idade máxima do táxon ou processos sucessionais, ou (ii) estocásticos, quando estão associados a existência de fogo, ventos muito intensos, temperaturas extremas, secas severas, inundações, poluição ou ataques de pragas (Pedersen 1998, Kitzberger et al. 2000). Em ecossistemas naturais é possível observar que algumas espécies são mais susceptíveis que outras a alguns desses fatores (Harcombe \& Marks 1983, Pedersen 1998).

Nesse contexto, os estudos de dendrocronologia podem ser utilizados para a análise de tendências demográficas, propiciando a compreensão da estrutura etária e de pulsos de recrutamento e mortalidade em espécies arbóreas com anéis de crescimento anuais (Pedersen 1998, Villalba \& Veblen 1998, Kitzberger et al. 2000, Suarez et al. 2004). Os anéis de crescimento anuais constituem verdadeiros bancos de dados naturais que podem armazenar informações ecológicas e históricas (Kozlowski et al. 1991, Kitzberger et al. 2000). Isso porque, o câmbio, tecido gerador das novas células de xilema e floema secundários, é considerado um sensor dos estímulos ambientais que afetam funções fisiológicas nas plantas. O câmbio responde às variações do ambiente e incorpora informações à estrutura dos anéis de crescimento (Schweingruber 1988, Kozlowski et al. 1991, Rubino \& McCarthy 2004).

Schizolobium parahyba apresenta anéis de crescimento distintos (Mainieri \& Chimelo 1989, Tomazello Filho et al. 2004, Lisi et al. 2008, Marcati et al. 2008). As investigações sobre a periodicidade de formação desses anéis revelaram o caráter anual dos mesmos em indivíduos crescendo em Floresta Estacional Semidecidual, no Estado de São Paulo (Ferreira 2002, Lisi et al. 2008, Marcati et al. 2008), e em Floresta Ombrófila Densa, no Estado do Rio de Janeiro (C.S. Lisi, dados não publicados).

Este trabalho tem por objetivo investigar, por meio dos anéis de crescimento, a idade de indivíduos mortos de S. parahyba na Ilha Grande, Rio de Janeiro, a relação desses anéis com os índices pluviométricos locais e a possível relação entre o episódio de mortalidade com processos etários e sucessionais.

\section{Material e métodos}

A Ilha Grande está situada no litoral sul do Estado do Rio de Janeiro e integra o Município de Angra dos Reis. É a terceira maior ilha brasileira, com uma área de $190 \mathrm{~km}^{2}$ e possui diferentes fitofisionomias da Floresta Atlântica, predominando a Floresta Ombrófila Densa (Oliveira \& Coelho Netto 2001). O clima da região é quente e úmido, com temperatura média anual de $24{ }^{\circ} \mathrm{C}$ (Oliveira \& Coelho Netto 2001). As médias de temperatura máxima podem ser superiores a $30^{\circ} \mathrm{C}$, em fevereiro, e as de temperatura mínima, inferiores a $10^{\circ} \mathrm{C}$, em julho (Salgado \& Vasquez 2009). A precipitação total anual alcança níveis superiores a $2.000 \mathrm{~mm}$ (Salgado \& Vasquez 2009). No período analisado, 1995 a 2001, a precipitação total média foi de $2.076 \mathrm{~mm}$, sendo o ano de 1996, o mais chuvoso $(2.515 \mathrm{~mm})$ e o ano de 1997 , o mais seco $(1.728 \mathrm{~mm})$, segundo dados obtidos da estação meteorológica da Central Nuclear Almirante Álvaro Alberto em Angra dos Reis/RJ.

A pesquisa foi desenvolvida na Vila do Abraão $\left(23^{\circ} 08^{\prime} 37^{\prime}\right.$ ' S- $\left.44^{\circ} 09^{\prime} 55,8^{\prime \prime} \mathrm{W}\right)$ e em três sítios, distribuídos em um raio de $10 \mathrm{~km}$ (tabela 1). Os sítios de coleta estavam localizados na zona de amortecimento do Parque Estadual da Ilha Grande, em trechos anteriormente ocupados para cultivos de subsistência e abandonados entre 10 e 25 anos, de acordo com Oliveira (2002). A altitude variou entre 20 e $80 \mathrm{~m}$ e o lençol freático esteve entre dois e três metros de profundidade nos pontos de coleta. As árvores selecionadas para o estudo morreram no período entre novembro de 2001 e março de 2002. Os discos amostrais, obtidos dessas árvores que não estavam tombadas, foram coletados a $1,30 \mathrm{~m}$ acima da superfície do solo, após 12 meses deste episódio de mortalidade.

Os indivíduos amostrados apresentavam diâmetros variados (tabela 1), fustes retos, sem bifurcações ou defeitos aparentes. Os discos amostrais foram secos à temperatura ambiente e polidos com lixas sucessivas de 36 a 400 grãos $\mathrm{cm}^{-2}$. Devido ao alto grau de deterioração da madeira e à existência de indivíduos com fustes inclinados, bifurcados ou com caules fusionados, apenas cinco indivíduos foram analisados. O material botânico foi depositado na Xiloteca do Instituto de Pesquisas Jardim Botânico do Rio de Janeiro (tabela 1). 
Tabela 1. Indivíduos amostrados, sítios de coleta (sítios: 1 = Praia do Canto; 2 = Assembléia e 3 = Serrado), número de registro na Xiloteca do Instituto de Pesquisa Jardim Botânico do Rio de Janeiro (RBw), diâmetro à altura do peito (DAP), número de anéis de crescimento (Anéis) e taxas de incremento radial (TI).

Table 1. Sampled trees, sites of collection (sítios: $1=$ Praia do Canto; 2 =Assembléia e 3 = Serrado), number of register in the wood collection of the Rio de Janeiro Botanical Garden (RBw), diameter at breast height (DAP), number of growth-rings (Anéis), and radial increment rates (TI).

\begin{tabular}{cccccc}
\hline Indivíduos & RBw & Sítios & $\begin{array}{c}\text { DAP } \\
(\mathrm{cm})\end{array}$ & Anéis & $\begin{array}{c}\text { TI } \\
\left(\mathrm{mm} \mathrm{ano}^{-1}\right)\end{array}$ \\
\hline 1 & 9042 & 1 & 49,0 & 53 & 4,4 \\
2 & 9043 & 2 & 40,1 & 33 & 19,0 \\
3 & 9044 & 3 & 24,5 & 39 & 9,0 \\
4 & 9045 & 3 & 22,6 & 35 & 13,0 \\
5 & 9046 & 2 & 78,6 & 69 & 10,2 \\
\hline
\end{tabular}

Para a descrição microscópica dos anéis de crescimento foram confeccionados corpos de prova do cerne da madeira (Coradin \& Muñiz 1991), que após 30 minutos de amolecimento em autoclave, foram seccionados ao micrótomo de deslizamento, nos planos transversal e longitudinal tangencial e radial, com espessuras entre 16 e $20 \mu \mathrm{m}$. As secções histológicas obtidas foram coradas pela solução aquosa de safranina $1 \%$ e azul de astra $1 \%$, na proporção $6: 4$
(Bukatsch 1972) e montadas em lâminas permanentes com resina sintética. As imagens foram obtidas utilizando câmera de vídeo Q Collor R3 acoplada ao microscópio Olympus BX41-BF-I-20. A imagem macroscópica foi obtida com câmera digital Sony DSC-40.

Para contagem do número de camadas de crescimento foram definidos dois a três raios em cada disco amostral, partindo da medula em direção ao córtex, no local em que os anéis apresentavam-se mais evidentes. Os raios de cada amostra foram confrontados e as camadas de crescimento descontínuas, descartadas. A idade dos indivíduos foi estimada em função do número de anéis de crescimento. Após datação dessas camadas, foi mensurada a largura dos anéis correspondentes aos anos de 1995 a 2001. A mensuração foi realizada sob microscópio estereoscópico e com auxílio de paquímetro digital. Em seguida, foi realizada a datação cruzada entre os anéis de crescimento das árvores amostradas (Stokes \& Smiley 1996). O teste de Spearman (Zar 1999) foi aplicado para correlacionar a largura dos anéis de crescimento de $S$. parahyba aos índices pluviométricos anuais.

\section{Resultados e discussão}

Schizolobium parahyba é uma espécie que apresenta ritmo periódico anual na fenologia e na formação dos anéis de crescimento (Ferreira 2002, Lisi et al. 2008, Marcati et al. 2008, C.S. Lisi, dados não publicados). Nos indivíduos estudados, esses anéis de crescimento são distintos macroscopicamente (figura 1A) e, estruturalmente (figura 1B), se caracterizam pelo achatamento radial das fibras

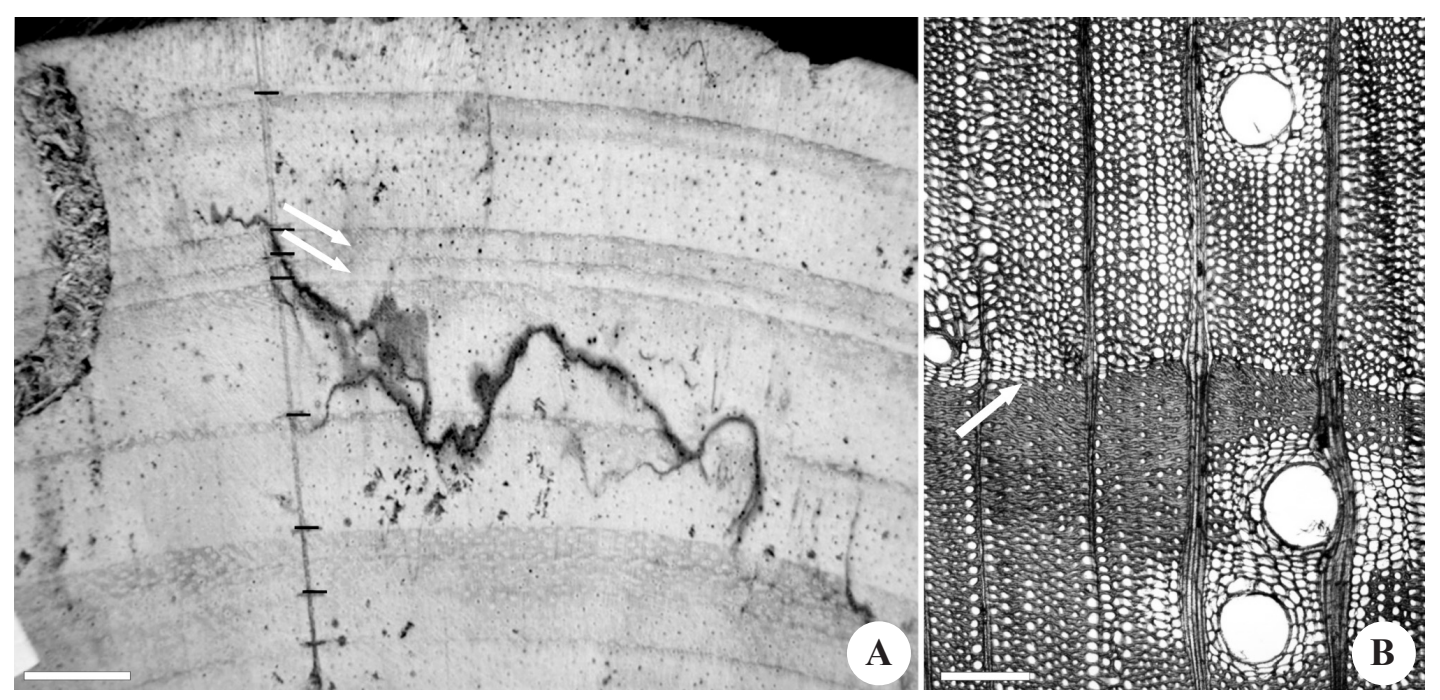

Figura 1. Secções transversais do lenho de Schizolobium parahyba. A. Anéis de crescimento do indivíduo quatro. Note os anéis de crescimento mais estreitos correspondentes aos anos de 1998 e 1999 (setas). B. Limite do anel de crescimento (seta). Barra $=0,6 \mathrm{~cm}(\mathrm{~A}) ; 200 \mu \mathrm{m}(\mathrm{B})$.

Figure 1. Transverse sections of Schizolobium parahyba wood. A. Growth-rings in tree number four. Note the narrow growth-rings corresponding to the years 1998 and 1999 (arrows). B. Growth-ring limit (arrow). Bar =0.6 cm (A), $200 \mu \mathrm{m}(\mathrm{B})$. 
e maior espessamento de suas paredes no lenho tardio e pela presença de parênquima em faixa marginal, com uma a três células de largura, repetindo o padrão referido na literatura (Ferreira 2002, Tomazello Filho et al. 2004, Lisi et al. 2008, Marcati et al. 2008).

$\mathrm{O}$ número de anéis de crescimento variou entre os indivíduos estudados, como pode ser observado na tabela 1 . O indivíduo 2, com 33 anéis de crescimento foi determinado como o mais jovem e o indivíduo 5, com 69 anéis, como o mais velho, ambos coletados no sítio 2 (tabela 1). A contagem do número de anéis anuais de crescimento da medula ao córtex proporciona a estimativa da idade da árvore na altura da amostra coletada, o que não, necessariamente, coincide com a idade total da árvore desde o momento de sua germinação (Kitzberger et al. 2000). Os resultados obtidos a partir das amostras estudadas neste trabalho constituem aproximações. A existência de falsos anéis ou de flutuações intra-anuais não é descartada totalmente, assim como a possibilidade das idades reais serem mais avançadas que as aqui relatadas. A existência de indivíduos com 69 anéis anos ${ }^{-1}$, a 1,30 $\mathrm{m}$ acima do solo, supera a longevidade potencial do táxon, que de acordo com informações provenientes de áreas de plantio e do conhecimento tradicional, está entre 40 e 50 anos (Lorenzi 1992).

A contagem e a datação dos anéis de crescimento demonstraram que os indivíduos estudados germinaram e alcançaram etapas de maturidade em escala temporal distinta, entretanto, a mortalidade ocorreu de forma sincrônica. $\mathrm{O}$ fato da mortalidade atingir indivíduos de meia idade, 33 anos, e bem maduros, 69 anos, exclui a possibilidade do evento representar a dinâmica particular de uma população senescente e, consequentemente, não pode ser considerada como um efeito demográfico relativo à idade máxima do táxon.

Schizolobium parahyba é uma espécie pioneira (Lorenzi 1992, Ferreira 2002, Oliveira 2002, Lisi et al. 2008) e os três sítios de coleta na Vila do Abraão foram determinados como em início de sucessão natural (Callado et al. 2009). As áreas são de transição entre a vegetação antropizada e a melhor preservada, constituindo áreas abertas, onde a luz incide diretamente no solo, descartando a possibilidade de morte em função do processo de regeneração da mata por sucessão natural.

A largura dos anéis de crescimento em cada ano avaliado refletiu uma taxa média de $11,1 \mathrm{~mm} \mathrm{ano}^{-1} \mathrm{de}$ incremento radial para os indivíduos de $S$. parahyba (tabela 1); resultado semelhante, $10,4 \mathrm{~mm}^{2} \mathrm{ano}^{-1}$, foi encontrado para espécie crescendo em outro sítio de Floresta Ombrófila Densa no estado do Rio de Janeiro (C.S. Lisi, dados não publicados). A análise da variação da largura dos anéis de crescimento apresentou correlação significativa com os índices pluviométricos anuais na área de estudo $\left(P<0,005\right.$ e $\left.r^{2}=0,390\right)$. A precipitação é o principal fator que atua na largura dos anéis de crescimento. Esses, de modo geral, são estreitos nos anos mais secos e mais largos nos anos mais chuvosos (Stokes \& Smiley 1996, Enquist \& Leffler 2001). Os anéis de crescimento formados nos anos de 1998 e 1999 foram mais estreitos que dos outros anos avaliados, apresentando $8 \mathrm{~mm}$ de largura em média (figura 2A-B). Esses anéis estreitos refletem um baixo crescimento radial e se formaram sob influência do evento El Niño dos anos 1997-1998, considerado o mais importante do século, segundo a cronologia de intensidade, e o de maior influência na região sudeste do Brasil, originando drásticas anomalias na distribuição de chuvas (Rao et al. 2001, Minuzzi et al. 2005). Nesse período, a precipitação total média foi de $1.750 \mathrm{~mm}$ ano-1 na região de estudo. Os anéis de crescimento formados antes e depois do período em questão apresentaram-se mais largos, com 15,4 mm de largura em média (figura 2A-B). Os dois últimos anéis de crescimento foram formados sob influência do fenômeno La Niña. Embora de intensidade moderada, o evento climático foi de longa duração, trazendo significativa elevação da umidade para o período (Rao et al. 2001), e registrado na região por uma precipitação total média de $2.115 \mathrm{~mm}$ ano $^{-1}$. A pluviosidade na bacia hidrográfica de Ilha Grande é uma das mais altas no território brasileiro (Davis \& Naghettini 2001, Salgado et al. 2007). A Serra do Mar constitui uma barreira orográfica de extrema importância para a elevada precipitação observada nesta região. Os fatores latitude e proximidade do mar criam condições de forte radiação solar, fornecimento de umidade e núcleos de condensação que, conjugados à posição da Serra em relação à trajetória dos sistemas frontais e linhas de instabilidade, provocam elevada precipitação (Davis \& Naghettini 2001). As chuvas intensas são uma característica climática marcante da região e podem promover saturação do solo, enxurradas e desencadear movimentos de massa (deslizamentos, corrida de lama, rastejo) mesmo em áreas vegetadas (Oliveira \& Coelho Neto 2001, Salgado \& Vasquez 2009).

A alternância brusca entre períodos muitos secos e úmidos pode promover desgaste fisiológico nos organismos vegetais. Segundo Keeland \& Sharitz (1997), a alternância no status hídrico do solo resulta em ciclos de morte e regeneração do sistema radicular, afetando consideravelmente o vigor das plantas arbóreas. Suarez et al. (2004) enfatizam o papel cumulativo das alterações do clima sobre as taxas de mortalidade de 

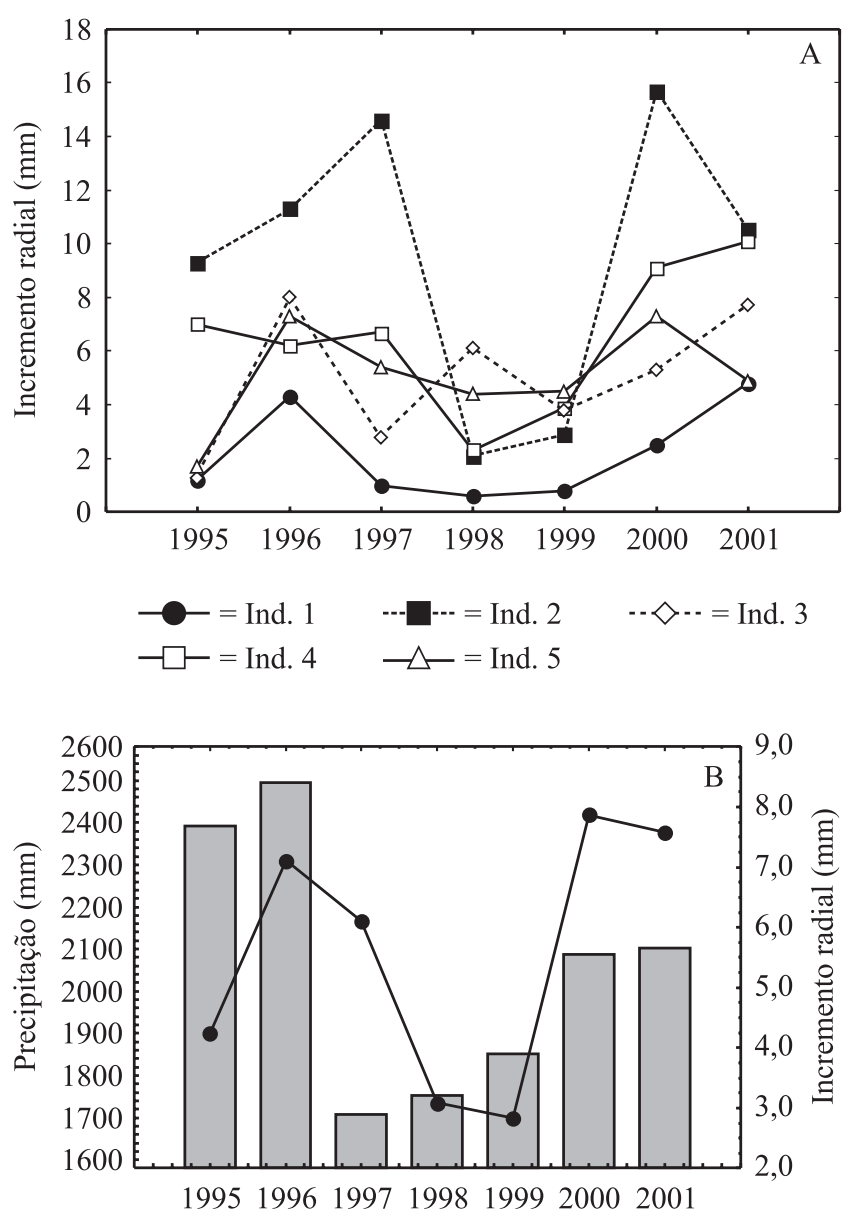

Figura 2. A. Incrementos radiais anuais dos indivíduos de $S$ Schizolobium parahyba estudados no período de 1995 a 2001. B. Precipitação anual e médias dos incrementos radiais.

Figure 2. A. Annual radial increments of the studied individuals of Schizolobium parahyba from 1995 to 2001. B. Annual precipitation and means of radial increment.

espécies em ambiente natural. As variações climáticas também podem aumentar ou reduzir a vulnerabilidade das árvores ao ataque de pragas oportunistas (Larsson 1989, Ryerson et al. 2003), como a de alguns insetos, cujas populações podem aumentar durante períodos mais quentes e úmidos (Hard et al. 1980, Campbell 1989, Ryerson et al. 2003, Veblen et al. 2007). Para o gênero Schizolobium Vogel existem relatos que a umidade elevada pode interferir na fitossanidade das árvores e aumentar a susceptibilidade ao surgimento de pragas (Sousa et al. 2005, Costa et al. 2006). Alguns patógenos podem atacar exclusivamente as espécies do gênero (Sousa et al. 2005, Trindade et al. 1999). As principais pragas citadas são: broca-damadeira (Acanthoderes jaspidea Germar), coleobroca (Micrapate brasiliensis Lesne), serradores (Oncideres dejeani Thompson e O. saga Dalma), mosca-damadeira (Rhaphiorhynchus pictus Wied.) e crostanegra-das-folhas (Phyllachora schizolobiicola subsp. schizolobiicola Henn.) (Richter et al. 1974, Sousa et al. 2005). Assim, não é possível descartar que patógenos possam ter se desenvolvido no longo período chuvoso, ocasionado pelo evento La Niña, e atacado os indivíduos de $S$. parahyba em Ilha Grande. Nenhuma constatação da existência de pragas foi realizada nos sítios de estudo no momento da obtenção das amostras de madeira. Contudo, experimentos realizados com indivíduos jovens de $S$. parahyba submetidos a crescimento sob solo alagado demonstraram a ocorrência de senescência e abscisão foliar, redução nos teores de clorofilas a e b e aparecimento de fungos nos ápices caulinares, ocasionando elevado índice de mortalidade, caracterizando a espécie como intolerante à inundação (Costa et al. 2006).

A mortalidade de espécies florestais relacionadas aos eventos El Niño e La Niña tem sido investigada com base em estudos dendrocronológicos (Pedersen 1998, Villalba \& Veblen 1998, Suarez et al. 2004) e relacionam os períodos severos de seca, resultantes desses fenômenos climáticos, à supressão das taxas de crescimento radial e à morte dos indivíduos arbóreos. Inúmeros estudos enfatizam o declínio do vigor, identificado no lenho pela redução das taxas de crescimento radial, como uma característica proeminente de árvores que estão morrendo (por exemplo, Pedersen 1998, Kitzberger et al. 2000, Cherubini et al. 2002, Suarez et al. 2004). Ao contrário, em $S$. parahyba, a largura dos anéis de crescimento evidencia taxas elevadas de crescimento no período que precede a morte dos indivíduos estudados, ou seja, no período que inclusive tem a influência do evento La Niña. No entanto, até o momento, nenhum estudo com base nos anéis de crescimento foi conduzido no sentido de relacionar a elevação dos índices pluviométricos a episódios de mortalidade. Tais estudos são necessários, especialmente nos trópicos, onde o período chuvoso ocorre normalmente no verão, quando as plantas apresentam taxas metabólicas mais elevadas e crescimento mais vigoroso (Pimenta et al. 1998, Lobo \& Joly 2000), como o que ocorreu na Ilha Grande com S. parahyba.

Agradecimentos - Ao Centro de Estudos Ambientais e Desenvolvimento Sustentável (Ceads), pela infraestrutura de trabalho disponibilizada na Ilha Grande; À Fundação Carlos Chagas Filho de Amparo à Pesquisa do Estado do Rio de Janeiro (Faperj), pelo apoio financeiro, ao Instituto Estadual de Floresta do Rio de Janeiro (IEF-RJ), atual, Instituto Estadual do Ambiente (Inea), pela licença de 
pesquisa concedida; à MSc. Leonor Ribas de Andrade, pela apresentação do problema e o auxílio na localização dos indivíduos de $S$. parahyba; ao Dr. Kenny Tanizaki Fonseca e ao Dr. Sebastião José da Silva Neto, pelas coletas das amostras de madeira; à Bióloga Jeanne Alexandre Teixeira da Glória, pela confecção das lâminas; ao Dr. Luiz Sebastião Poltronieri, pelo envio de material bibliográfico e à Dra. Maura da Cunha, ao MSc. Gabriel U.C. Araújo dos Santos e aos dois assessores anônimos e editores deste periódico, pela leitura crítica, correções e sugestões a este trabalho.

\section{Referências bibliográficas}

BUKATSCH, F. 1972. Bermerkungen zur Doppelfärbung Astrablau-Safranin. Mikrokosmos 61:255.

CALLADO, C.H., BARROS, A.A.M. DE, ANDRADE, L.R. DE, ALBARELLO, N., GAGLIARDI, R., CASSANO, V. \& JASCONE, C.E. 2009. Flora e vegetação da Ilha Grande. In $\mathrm{O}$ ambiente da Ilha Grande (M.P. Bastos \& C.H. Callado, eds.). Universidade do Estado do Rio de Janeiro, Rio de Janeiro, p.93-146.

CAMPBELL, I.M. 1989. Does climate affect host-plant quality? Annual variation in the quality of balsamfir as food for spruce budworm. Oecologia 81:341-344.

CHERUBINI,P., FONTANA, G., RIGLING, D., DOBBERTIN, M., BRANG, P. \& INNES, J.L. 2002. Tree-life history prior to death: two fungal root pathogens affect tree-ring growth differently. Journal of Ecology 90:839-850.

CORADIN, V.T. \& MUÑIZ, G.M.B. 1991. Normas de procedimentos em estudos de anatomia de madeira: I. Angiospermae. II. Gimnospermae. Laboratório de Produtos Florestais 15:1-19.

COSTA, A.M., GOBBI, E.L., DEMUNER, V.G. \& HEBLING, S.A. 2006. O efeito da inundação do solo sobre o crescimento inicial de Schizolobium parahyba (Vell.) S.F. Blake, guapuruvu. Natureza on line 4:7-13.

DAVIS, E.G. \& NAGHETTINI, M.C. 2001. Estudo de chuvas intensas no Estado do Rio de Janeiro. CPRM, Belo Horizonte.

ELLSTRAND, N.C. \& ELAM, D.R. 1993. Population genetic consequences of small population size: implications for plant conservation. Annual Review of Ecology and Systematics 24:217-242.

ENQUIST, B.J. \& LEFFLER, A.J. 2001. Long-term tree ring chronologies from sympatric tropical dry-forest trees: individualistic responses to climatic variation. Journal of Tropical Ecology 17:41-60.

FERREIRA, L. 2002. Periodicidade do crescimento e formação da madeira de algumas espécies arbóreas de Florestas Estacionais Semidecíduas da região sudeste do Estado de São Paulo. Dissertação de mestrado, Universidade de São Paulo, ESALQ, Piracicaba.

FRANKLIN, J.F., SHUGART, H.H. \& HARMON, M.E. 1987. Tree death as an ecological process. BioScience $37: 550-556$.
FREIRE, J.M., PIÑA-RODRIGUES, F.C.M., LIMA, E.R. DE, SODRÉ, S.R.C. \& CORRÊA, R.X. 2007. Estrutura genética de populações de Schizolobium parahyba (Vell.) Blake (guapuruvu) por meio de marcadores RAPD. Scientia Forestalis 74:27-35.

HARCOMBE, P.A. \& MARKS, P.L. 1983. Five years of tree death in a Fagus-Magnolia forest, southeast Texas (USA). Oecologia 57:49-54.

HARD, J., TUNNOCK, S. \& EDER, R. 1980. Western spruce budworm defoliation trend relative to weather in the Northern Region, 1969-1979. US Department of Agriculture, Forest Services, Northern Region, State and Private Forestry Report, Missoula.

INOUE, M.T., RODERJAN, C.V. \& KUNIYOSHI, Y.S. 1984. Projeto Madeira do Paraná. Fundação de Pesquisas Florestais do Paraná, Curitiba.

KEELAND, B.D. \& SHARITZ, R.R. 1997. The effects of water-level fluctuations on weekly tree growth in a Southeastern USA swamp. American Journal of Botany 84:131-139.

KITZBERGER, T., VEBLEN, T.T. \& VILLALBA, R. 2000. Métodos dendroecológicos y sus aplicaciones en estudios de dinámica de bosques templados de Sudamérica. In Dendrocronología en América Latina (F.A. Roig, ed.). Ediunc, Mendoza, p.17-78.

KOZLOWSKI, T.T., KRAMER, P.J. \& PALLARDY, S.G. 1991. The physiological ecology of woody plants. Academic Press, San Diego.

LARSSON, S. 1989. Stressful times for the plant stress insect performance hypothesis. Oikos 56:277-283.

LISI, C.S., TOMAZELLO-FILHO, M., BOTOSSO, P.C., ROIG, F.A., MARIA, V.R.B., FERREIRA-FEDELE, L. \& VOIGT, A.R.A. 2008. Tree-ring formation, radial increment periodicity and phenology of tree species from a Seasonal Semi-Deciduous Forest in Southeast Brazil. IAWA Journal 29:189-207.

LOBO, P.C. \& JOLY, C.A. 2000. Aspectos ecofisiológicos da vegetação de mata ciliar do Sudeste do Brasil. In Matas ciliares: conservação e recuperação (R.R. Rodrigues \& H.F. Leitão Filho, eds.). Edusp, São Paulo, p.143-157.

LORENZI, H. 1992. Árvores Brasileiras. Instituto Plantarum, Nova Odessa.

MAINIERI, C. \& CHIMELO, J.P. 1989. Fichas de características das madeiras brasileiras. Instituto de Pesquisas Tecnológicas - IPT, São Paulo.

MARCATI, C.R., MILANEZ, C.R.D. \& MACHADO, S.R. 2008. Seasonal development of secondary xylem and phloem in Schizolobium parahyba (Vell.) Blake (Leguminosae: Caesalpinioideae). Trees 22:3-12.

MINUZZI, R.B., SEDIYAMA, G.C., RIBEIRO, A. \& COSTA, J.M.N. DA. 2005. El Niño: ocorrência e duração dos veranicos do Estado de Minas Gerais. Revista Brasileira de Engenharia Agrícola e Ambiental 9:364-371. 
OLIVEIRA, R.R. 2002. Ação antrópica e resultantes sobre a estrutura e composição da Mata Atlântica na Ilha Grande, RJ. Rodriguésia 53:33-58.

OLIVEIRA, R.R. \& COELHO NETTO, A.L. 2001. Captura de nutrientes atmosféricos pela vegetação na Ilha Grande, RJ. Pesquisa Botânica 51:31-49.

PEDERSEN, B. 1998. The role of stress in the mortality of midwestern oaks as indicated by growth prior to death. Ecology 79:79-93.

PIMENTA, J.A., BIANCHINI, E. \& MEDRI, M.E. 1998. Adaptations to flooding by tropical trees: morphological and anatomy modifications. Oecologia Brasiliensis 4:157-176.

PIO CORRÊA, M.P. 1926. Dicionário das plantas úteis do Brasil e das exóticas cultivadas. Ministério da Agricultura, Rio de Janeiro.

RAO, V.B., SANTO, C.E. \& FRANCHITO, S.H. 2001. A diagnosis of rainfall over South America during 1997/98 El Niño event. Part I: validation of NCEPNCAR reanalysis of rainfall data. Journal of Climate 15:502-511.

RICHTER, H.G., TOMASELLI, I. \& MORESCHI, J.C. 1974. Estudo tecnológico do guapuruvu (Schizolobium parahybum). Revista Floresta 5:26-30.

RICKLEFS, R. 2003. A economia da natureza. GuanabaraKoogan, Rio de Janeiro.

RIZZINI, C.T. 1995. Árvores e madeiras úteis do Brasil: manual de dendrologia brasileira. Edgard Blucher, São Paulo.

RUBINO, D.L. \& MCCARTHY, B.C. 2004. Comparative analysis of dendroecological methods used to assess disturbance events. Dendrochronologia 21:97-115.

RYERSON, D.E., SWETNAM, T.W. \& LYNCH, A.M. 2003. A tree-ring reconstruction of western spruce budwormoutbreaks in the San Juan Mountains, Colorado, USA. Canadian Journal of Forestry Research 33: 1010-1028.

SALGADO, C.M. \& VASQUEZ, N.D. 2009. Clima. In O ambiente da Ilha Grande (M.P. Bastos \& C.H. Callado, eds.).Universidade do Estado do Rio de Janeiro, Rio de Janeiro, p.7-20.
SALGADO, C.M., PEIXOTO, M.N.O. \& MOURA, J.R.S. 2007. Caracterização espaço-temporal da chuva como subsídio à análise de episódios de enchentes no município de Angra dos Reis, RJ. Geosul 22:7-26.

SANTOS, M.S. DOS, SANTIAGO, A.M.A., GUIMARÃES, C., NOGUEIRA, I. \& SANT'ANNA, T. 2009. História da Ilha Grande e patrimônio cultural material e imaterial. 2009. In O ambiente da Ilha Grande (M.P. Bastos \& C.H. Callado, eds.). Universidade do Estado do Rio de Janeiro, Rio de Janeiro, p.273-345.

SCHWEINGRUBER, F.H. 1988. Tree rings: basics and applications of dendrochronology. D. Reidel Publishing Company, Dordrecht.

SOUSA, D.B., CARVALHO, G.S. \& RAMOS, E.J.A. 2005. Paricá - Schizolobium amazonicum Huber ex Ducke. Informativo Técnico Rede de Sementes da Amazônia: 13. Rede de Sementes da Amazônia, Manaus.

STOKES, M.A. \& SMILEY, T.L. 1996. An introduction to tree-ring dating. The University of Arizona Press, Tucson.

SUAREZ, M.L., GHERMANDI, L. \& KITZBERGER, T. 2004. Factors predisposing episodic drought-induced tree mortality in Nothofagus - site, climatic sensitivity and growth trends. Journal of Ecology 92:954-966.

TOMAZELLO FILHO, M., LISI, C.S., HANSEN, N. \& CURY, G. 2004. Anatomical features of increment zones in different tree species in the state of São Paulo, Brazil. Scientia Forestalis 66:46-55.

TRINDADE, D.R., POLTRONIERI, L.S., BENCHIMOL, R.L., ALBUQUERQUE, F.C. \& OLIVEIRA, N.T. 1999. Black crust (Phyllachora schizolobiicola subsp. schizolobiicola) on Schizolobium amazonicum in Brazil. Fitopatologia Brasileira 24:194.

VEBLEN, T.T., YOUNG, K.R. \& ORME, A.R. 2007. The physical geography of South America. Oxford University Press, Oxford.

VILLALBA, R. \& VEBLEN, T.T. 1998. Influences of largescale climatic variability on episodic tree mortality in Northern Patagonia. Ecology 79:2624-2640.

ZAR, J.H. 1999. Biostatistical analysis. Prentice-Hall, New Jersey. 
